\title{
LS-SVM-based On-Load Tap Changer Control for Distribution Networks with Rooftop PV's
}

\author{
Sam Weckx, Carlos Gonzalez, Tom De Rybel and Johan Driesen \\ KULeuven Energyville \\ sam.weckx@esat.kuleuven.be
}

\begin{abstract}
This paper discusses voltage regulation using onload tap changers (OLTC) with line drop compensation (LDC) on low-voltage distribution networks with a high penetration of photovoltaic generation (PV). Load flow simulations show that PV generators affect the performance of classical LDC in a negative way. However, when a database exists where voltages measured by smart-meters are stored, Least-Squares support vector machines (LS-SVM) can be used to estimate the maximum and minimum voltage in the system, without needing a grid model, nor real-time communication. Control of the OLTC can then be executed to widen the gap between the system voltage and the acceptable limits, based on the maximum and minimum system voltage estimates.
\end{abstract}

Index Terms-Least-squares support vector machines, on-load tap changer, line drop compensation, photovoltaic generation

\section{INTRODUCTION}

High penetration of distributed photovoltaic generators in low-voltage distribution networks challenges future grid operation. Their introduction in the grid causes a reduction, or even a reversal, of the real power flow in the low voltagenetwork. A reverse power flow results in a voltage rise across a distribution feeder. Distribution System Operators (DSO) have to comply with national standards to keep the voltage within acceptable limits [1]. DSOs are interested in an economical solution with high reliability and controllability to cope with the increasing amount of PV generation. The solution must be applicable for a high variety of distribution grids, with possible unknown or non-digitized topology. An on-load tap changer (OLTC) can be an economical efficient solution when the additional costs for the OLTC and the necessary ICT are limited [2]. In this paper we propose a method to control the OLTC which does not rely on real-time communication and therefore has a high reliability and reduced cost for the ICT.

The tap changing of the OLTC is carried out in order to maintain the grid voltage between limits for all customers. The use of an OLTC can significantly increase the maximum amount of distributed generation that can be connected to the network without disrupting voltage regulation [3]. No LV OLTC are used today in LV grids in Flanders, however they are already commercially available [4]. Also in [5] an adapted LV substation equipped with OLTC was developed.

This research was supported by the EIT KIC INNOENERGY KIC-ASS project. S. Weckx has a PhD fellowship of the Research Foundation - Flanders - VITO (FWO-VITO)
Two classic voltage control methods for OLTCs exist [6]. The first method keeps the voltage at the LV side of the transformer constant. Tapping actions are performed by feeding-back the measured voltage at the secondary side of the transformer. Only voltage variations occurring in the MV voltage level and the voltage drop over the transformer are compensated. The other method is the line voltage drop compensator (LDC). A LDC measures the total current flow through the transformer and adapts the sending voltage based on a proportional relationship with the current flow. PV generators cause a reduction or reversal of this flow, resulting in a more complex voltage profile across the feeder. This might decrease the performance of the LDC. Therefore, it is often suggested to coordinate the LDC with the DG output to ensure proper voltage regulation [7]-[9]. Another approach is applied in [8] and [9]. Here, the voltage is measured at various points in the grid, to make it easier to control the voltage at other points in the grid, requiring a real-time communication channel. A full state estimation to optimally control the OLTC is applied in [10] for MV grids. This requires a complete grid model and real-time communication of measurements or pseudo-measurements.

This paper proposes a method to estimate, at the substation, the extreme states of a grid with a high penetration of PV. This is done based on a database of previous measured states, without having a network model, nor real-time communication. Based on the minimum and maximum voltage, the OLTC is controlled. The focus of this paper is on rooftop PV installations, as these are the strict majority of installed distributed generators in the Belgian low-voltage network.

\section{Simulated NETWORK}

The network used in simulations is a 3-phase 4-wire network and consists of an OLTC to which three different feeders are connected. The cables of feeder 1 and 3 are of type EXVB $1 \mathrm{kV} 4 \times 70 \mathrm{~mm}^{2}$. The cables of feeder 2 are of type EAXVB $1 \mathrm{kV} 4 \times 150 \mathrm{~mm}^{2}$ except for the first 50 meters of cable after the busbar, which is of type EAXVB $1 \mathrm{kV} 4 \times 95$ $\mathrm{mm}^{2}$. Cable properties are defined in table I. The impedance values are calculated according to design specifications in the Belgian standard for underground distribution cables NBN C33-322 [11] with an assumed operating temperature of $45^{\circ} \mathrm{C}$. Feeder 1 and 2 were found to be vulnerable for power quality problems with increasing amounts of PV generation [12]. These are existing feeders in Flanders and are connected to 


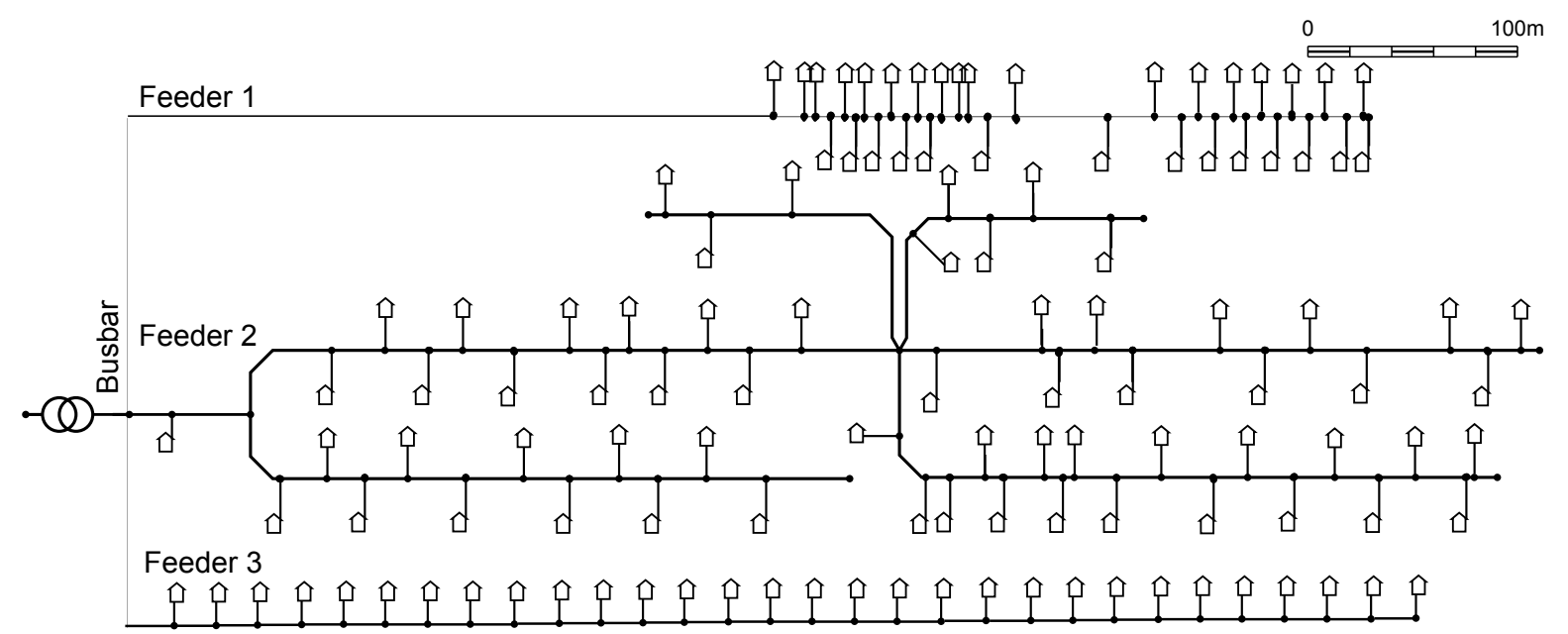

Fig. 1. The network used in the simulations. All lengths are drawn on scale.

TABLE I

CABLE PROPERTIES OF THE SIMULATED NETWORK

\begin{tabular}{l|r|l} 
Properties & Value & Unit \\
\hline \hline Impedance of EXVB 1 kV $4 \times 70 \mathrm{~mm}^{2}$ & $0.268+0.078 \mathrm{j}$ & {$[\Omega / \mathrm{km}]$} \\
Impedance of EAXVB 1 kV $4 \times 95 \mathrm{~mm}^{2}$ & $0.352+0.078 \mathrm{j}$ & {$[\Omega / \mathrm{km}]$} \\
Impedance of EAXVB 1 kV $4 \times 150 \mathrm{~mm}^{2}$ & $0.227+0.078 \mathrm{j}$ & {$[\Omega / \mathrm{km}]$}
\end{tabular}

one substation to obtain a representative network. In feeder 1 and 2 all households have PV generation, whereas in feeder 3 only half of the houses have rooftop PV panels installed. As the geographical area was small, the power output of all PV installations relative to their rated capacity was considered as being equal. The PV operated at unity power factor. All households have a single-phase connection with a nominal line-to-neutral voltage of $230 \mathrm{~V}$ and are equally spread across the three phases. The voltage at the primary side of the transformer is assumed to be 1 p.u. Statistically representative residential load profiles are available to perform load flow simulations [13]. The voltage is limited to $\pm 10 \%$ of the nominal voltage. In this research, it is assumed that smart meters are widespread throughout the grid, and are able to measure voltage. The DSO, or a third party, is responsible to collect the data regularly (i.e. monthly) and store them in a database. The DSO can access the values in the database. There is no real-time communication needed between the smart-meters and the DSO, reducing cost and complexity. The substation is equipped with a current transformer on each feeder.

\section{LINE DROP COMPENSATION (LDC)}

\section{A. Classical LDC voltage regulation}

The classical LDC method estimates the magnitude of the voltage at an end customer, and controls the OLTC to compensate the voltage. The estimated voltages are calculated by [6]

$$
V_{r}=V-(R+j X) I
$$

Where $V_{r}$ is the end customer voltage, $V$ is the sending voltage, $I$ the current measured at the beginning of the feeder in the substation and $(R+j X)$ the equivalent impedance of the distribution line (or the relay compounding settings [14]). By adapting the equivalent impedance, the estimation of the voltage is controlled. However PV generators reduce or reverse the active power flow, making the measured feeder currents no longer proportional to the load current. This way, the customer end voltage might be wrongly estimated.

\section{B. Influence of PV location}

The location where the PV generators are installed across the feeder significantly influences the voltage profile. When all PV generators are installed at the end of the feeder, the impedance faced by the generated power is higher than when installed in the beginning of the feeder, and so the voltage rise over it as well. This is shown in Fig. 2, where the voltage of an end customer is simulated as a function of the active current measured at the substation for a typical week in spring. The simulated feeder is feeder 3 of Fig. 2. 50\% of the houses in this feeder are equipped with a PV generator. In case all PV generators are installed at houses in the beginning of the feeder, the end customer voltage is clearly lower than when installed at the end of the feeder. The MV voltage level is kept constant in the simulation for generating this figure.

\section{Optimal Classical LDC settings}

It is clear from Fig. 2 that the relation between active current measured at the substation and the end customer voltage depends on the location of the PV installations. This results in a deteriorated performance of the classical LDC when this is not taken into account when defining the equivalent impedance of the distribution line. 


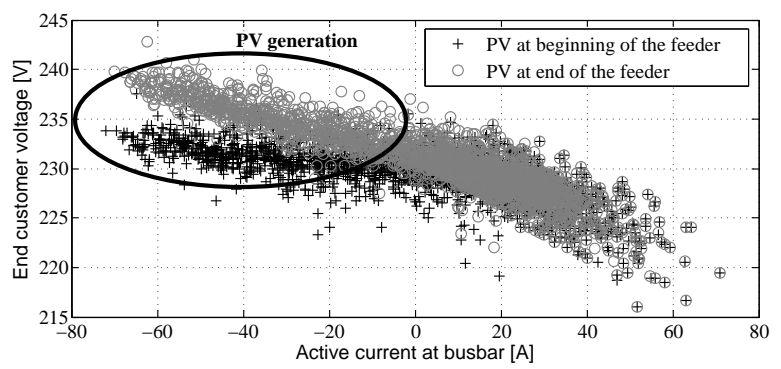

Fig. 2. End customer voltage as a function of the active current, for PV installed at the beginning and at the end of the feeder.

The voltage difference between the end customer voltage and the sending voltage can be approximated by [15]:

$$
\left|V_{r}\right|-|V| \approx-R I_{a c t}-X I_{\text {react }}
$$

When the smart-meter measured voltages are regularly read out and stored in a database, the optimal equivalent impedance of the classical LDC can be estimated in a linear least-squares sense. Which has as solution:

$$
\left[\begin{array}{l}
R \\
X
\end{array}\right]=\left(\left[I_{\text {act }} I_{\text {react }}\right]^{T}\left[I_{\text {act }} I_{\text {react }}\right]\right)^{-1}\left[I_{\text {act }} I_{\text {react }}\right]\left[|V|-\left|V_{r}\right|\right]
$$

Where $V_{r}$ is a vector containing the end customer voltages for a period, which was stored in the smart-meter database and accessed by the substation voltage estimator. $V$ is a stored vector of the sending voltages. $I_{\text {act }}$ and $I_{\text {react }}$ are stored vectors containing the measured active and reactive currents. $R$ and $X$ are the parameters to estimate. Note that when historical data is available, no knowledge about the grid is required to define the optimal equivalent impedance of the distribution line.

When expert knowledge is available, this can be incorporated in the identification of the optimal equivalent impedances by rewriting (3) as a constrained convex optimization problem. In case an expert has defined limits on the equivalent impedance and the $\frac{X}{R}$-ratio, the problem becomes:

$$
\begin{array}{ll}
\min _{R, X} & \left\|\left|V_{r}\right|-|V|+R I_{\text {act }}+X I_{\text {react }}\right\|^{2} \\
\text { subj. to } & R_{\text {min }} \leq R \leq R_{\max } \\
& X_{\min } \leq X \leq X_{\max } \\
& X=c R ;
\end{array}
$$

where $R_{\min }$ and $R_{\max }$ are the lower and upper bound on the equivalent resistance, $X_{\min }$ and $X_{\max }$ are the lower and upper bound on the equivalent inductance and $\mathrm{c}$ is the required $\frac{X}{R}$-ratio.

From the right side of Fig. 2, it is clear that when there is no generation, i.e. at the evening, the equivalent impedance is independent of the location of the installed PV, whereas during generation, the equivalent impedance depends on the location. Therefore, these parameters behave nonlinear. Besides that, in unbalanced 3-phase 4-wire grids, power consumption in one phase affects the phase voltages of the other phases due to the voltage drop over the neutral conductor [16]. Performance of the end customer voltage estimation will be limited when only the transformer current of the respective phase is taken into account to obtain the voltage estimation. A more accurate estimation of the end customer voltage can be done with nonlinear regression techniques, which take as input all the measured currents and time. Traditional least-squares do not map data in higher dimension spaces and assume linear behavior, hence LS-SVM can be used as a more general and flexible treatment on this regression problems.

\section{LS-SVM for the estimation of system voltages}

A LS-SVM is a supervised kernel based learning method that can be applied for regression [17]. Given a set of training data $\left(x_{i}, y_{i}\right)$, where $x_{i}$ are input vectors and $y_{i}$ are the corresponding output values, the least-squares support vector regression solves an optimization problem [17]:

$$
\begin{array}{ll}
\underset{w, b, e}{\operatorname{minimize}} & \frac{1}{2} w^{T} w+\gamma \sum_{i=1}^{n} e_{i}^{2} \\
\text { subject to } & y_{i}=w^{T} \varphi\left(x_{i}\right)+b+e_{i}
\end{array}
$$

Where $x_{i}$ is mapped to a higher dimensional space by the function $\varphi$. The parameters that control the regression quality are the cost of the error $\gamma$ and the mapping function $\varphi$. The inner product of the mapping functions is defined as the kernel. With the obtained LS-SVM parameters $w$ and $b$ or with the dual variables of the optimization problem the output for new inputs can be calculated [17]. The Radial Basis Function (RBF) kernel is applied, which has one controllable parameter. The applied LS-SVM has two tuning parameters to obtain a good regression quality: the cost of error and the RBF kernel parameter. Selection of the tuning parameters is done by cross-validation. The active and reactive current of all phases of all feeders are measured at the substation and are the input $x_{i}$ for the LS-SVMs. Time is also included in this paper as input, as most customers have a time-dependent and repetitive consumption profile. Their location across the feeder results in an influence of time on the relationship between the measured current and the end customer voltage. Also, the PV power output is clearly time-dependent, due to the location of the sun. Historical end customer voltage measurements are the corresponding output values $y_{i}$ used for the training of the LS-SVM. The solution of the optimization problem is obtained by the LS-SVMlab toolbox [18]. With the resulting LS-SVM parameters the estimated output voltages for new inputs can also be obtained with this toolbox.

Fig. 3 shows the end customer voltage for a typical spring day, together with the LS and LS-SVM estimate. The LS-SVM has as input the currents of all the three phases measured at the busbar, as well as the time, whereas the LS estimate only has the current measured at the busbar of the same phase as the end customer is connected to. The LS-SVM method has a $27 \%$ smaller sum squared error. So, even when the classical LDC settings are optimally chosen in a LS sense, a LS-SVM 


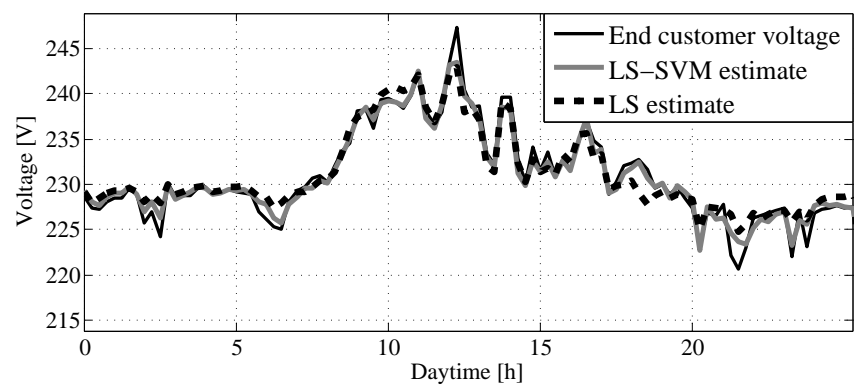

Fig. 3. Estimate of the end customer voltage by LS and LS-SVM.

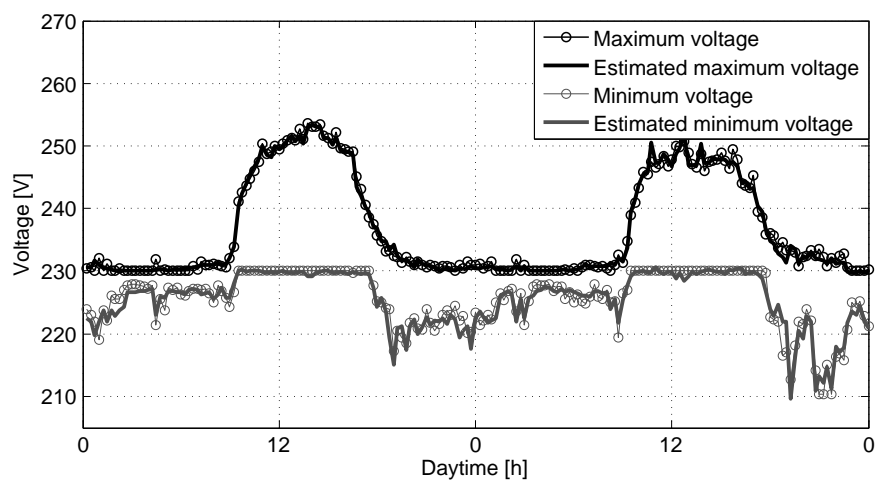

Fig. 4. System maximum and minimum voltage estimate by two LS-SVMs.

results in a better estimation of the end customer voltage. The LS and LS-SVM are trained based on data of the two previous months.

LDC regulation is more complicated when multiple feeders controlled by one LDC are loaded differently. With distributed generation, it is not sure that the end nodes are facing the maximum or minimum voltages. When controlling the OLTC, what is of interest is the maximal and minimal voltage occurring in all feeders. Therefore it is preferred to estimate the maximum and minimum voltage across all feeders instead of estimating the end-customer voltage. The maximum and minimum of the whole system could be estimated by only 2 LS-SVMs. Fig. 4 compares the estimates of the maximum and minimum voltage with the actual values. The LS-SVMs are trained based on the data of the month before.

\section{Control of The OLTC}

Based on the obtained estimates of minimum and maximum voltage occurring in the system, the optimal tap can be selected. The purpose of the tapping in this paper is to maximize the gap with the upper and lower limit of the allowable voltage. For example, when the minimal voltage in the system is close to the lower allowed limit, whereas the maximum voltage in the system has a big margin towards the upper limit, the sending voltage should be increased. Therefore, tapping is done based on the following formulas:

Tap up when:

$$
\text { Margin }_{\text {high }}-\text { Margin }_{\text {low }} \geq B W . \triangle \text { tap }_{\text {high }}
$$

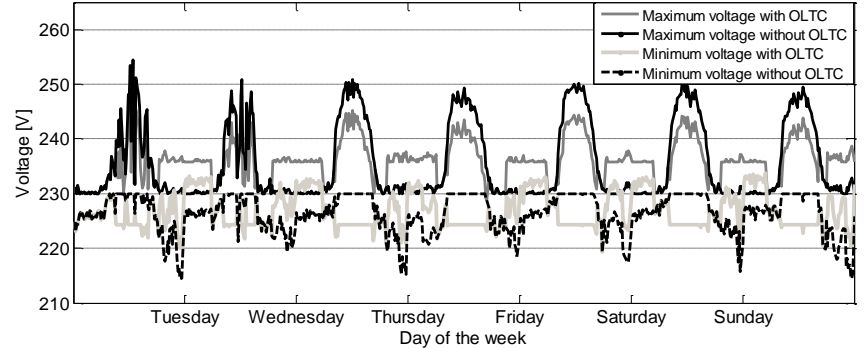

Fig. 5. Maximum and minimum voltage in the system with and without OLTC.

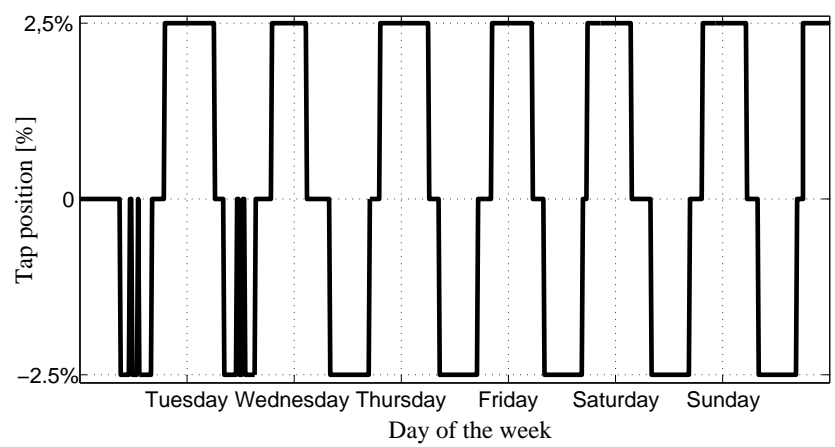

Fig. 6. Tap position.

Tap down when:

$$
\text { Margin }_{\text {low }}-\text { Margin }_{\text {high }} \geq B W . \triangle \text { tap }_{\text {low }}
$$

Where Margin $_{\text {high }}$ is the margin between the estimated maximum system voltage and the allowed upper limit [1], Margin $_{\text {low }}$ is the margin between the estimated minimum system voltage and the allowed lower limit, $\triangle t_{\text {tap }}$ high is the voltage difference between the actual tap and a higher tap, and $\triangle t_{\text {taplow }}$ is the voltage difference between the actual tap and a lower tap. The amount of tapping can be controlled by the bandwidth $B W$. Preferably, Margin high and Margin low $_{\text {low }}$ are equal, as this maximizes the gap with the limits. When the difference between the two margins becomes larger than the voltage difference between two taps, changing the tap will widen the gap with the limits.

By increasing the bandwidth, the amount of tapping will be reduced. In this simulation the OLTC is equipped with 3 taps of $230 \mathrm{~V} \pm 2.5 \%$. This is sufficient to avoid voltages rising or dropping to critical levels of $230 \mathrm{~V} \pm 10 \%$. The LS-SVMs are trained based on data from the two previous months, the bandwidth is chosen equal to a factor of 1.5 as this sufficiently avoids frequent tapping. Fig. 5 presents the maximum and mimimum system voltage with and without the OLTC for a simulation of one week. In Fig. 6 the according tap position is shown.

The optimal tap is not selected based on system losses. The influence of a voltage change at the secondary of the transformer on the system losses depends on the assumptions made. Resistive loads will consume more power when the 
voltage increases and so will create more losses. When the voltage increases in case of constant power loads or generators, the losses will reduce due to smaller currents.

\section{Advantages}

The main advantage of the proposed algorithm is the limited amount of information necessary for the operation of the OLTC. Grid topology information is not required for this algorithm. No real-time information needs to be communicated. Local measurements in the substation are sufficient. The LSSVM estimates the system minimum and maximum voltage and therefore takes into account different feeders that might behave different. Regularly (i.e. monthly), the LS-SVM should be retrained based on the stored data, to cope with changes in consumer and generator behaviour due to seasonal effects. Only at this moment the substation should communicate with a time-stamped database where the voltages measured by the smart meters are stored. Limitation of the communication requirements is crucial for a successful integration of OLTC in the distribution network, as the economic efficiency of the OLTC for the distribution transformer is strongly dependent on the additional costs for the necessary ICT [2].

One of the other main concerns in smart grids is the privacy of the customers [19]. Power signals expose customer habits and behaviors. As only the voltage at the point of connection is required, privacy of the customers is guaranteed. Besides that, it is not required that the voltage logger of the customer is continuously connected over a vast network. The data can be send in a batch. This reduces the vulnerability for malicious hackers. Finally, as the system is not dependent on real-time communication, the reliability is increased.

Another application of this research can be as a backup of a real-time communication-based system that has failed, while the system still needs an estimation of its state at the substation to perform tapping of an OLTC or to take other measures.

For this algorithm to work properly, some requirements should be met. First of all, currents and voltages need to be measured in each phase of each feeder at the substation, while smart meters measure the voltage throughout the grid. An important issue is the time delay between the measurements taken at the different locations. All these measurements in the LV grid should be time stamped. It is, however, not necessary to have information of the location or the phase of the smart meter. Only the system minimum and maximum voltage measured needs to be stored in a database, together with the measured currents and voltages at the substation. This reduces the required database capacity.

\section{CONCLUSION}

Performance of classical LDC with an OLTC is reduced by PV generators. When a database of measured voltages is available, the LDC settings can be defined by least squares. LS-SVM generally performs better in estimating voltages in a feeder. This paper proposes to use two LS-SVMs to estimate both maximum and minimum voltage across a low voltage network. This estimate is used to widen the margin to the voltage limits by tapping the transformer. Simulations show that controlling an OLTC based on estimates of LS-SVMs will significantly improve the margin towards the voltage limits, without requiring any information about the grid topology or real-time communication. Furthermore this concept has important end-user privacy advantages.

\section{REFERENCES}

[1] NEN-EN50160, "Voltage characteristics of electricity supplied by public electricity networks," 2010.

[2] T. Stetz, F. Marten, and M. Braun, "Improved low voltage gridintegration of photovoltaic systems in germany," Sustainable Energy, IEEE Transactions on, vol. PP, no. 99, pp. 1 -9, 2012.

[3] Ferry A. Viawan, Ambra Sannino, and Jaap Daalder, "Voltage control with on-load tap changers in medium voltage feeders in presence of distributed generation," Electric Power Systems Research, vol. 77, no. 10 , pp. $1314-1322,2007$

[4] Reinhausen (Jul 2013), "The voltage regulated gridcon transformer," Available:http://www.reinhausen.com/desktopdefault.aspx/tabid-1516/ 1835 read- 4652.

[5] J. Kester, P. Heskes, S. Kaandorp, J. Cobben, G. Schoonberg, D. Malyna, E. De Jong, B. Wargers, and A. Dalmeijer, "A smart MV/LV-station that improves power quality, reliability and substation load profile," in CIRED, 20th International Conference on Electricity Distribution, 2003.

[6] A. Uchida, S. Watanabe, and S. Iwamoto, "A voltage control strategy for distribution networks with dispersed generations," in Proc. IEEE Power Engineering Society General Meeting, 2007, pp. 1-6.

[7] J. Choi and J. Kim, "Advanced voltage regulation method of power distribution systems interconnected with dispersed storage and generation systems," IEEE Transactions on Power Delivery, vol. 16, no. 2, pp. 329-334, 2001.

[8] H. Leite, N. Jenkins, and P. Gale, "Real-time voltage regulation of distribution networks with distributed generation," in CIRED, 17th International Conference on Electricity Distribution, 2003.

[9] P. Kadurek, J. Cobben, and W. Kling, "Smart MV/LV transformer for future grids," in Proc. Int Power Electronics Electrical Drives Automation and Motion (SPEEDAM) Symp, 2010, pp. 1700-1705.

[10] M. Hird, N. Jenkins, and P. Taylor, "An active $11 \mathrm{kV}$ voltage controller: Practical considerations," in CIRED, 17th International Conference on Electricity Distribution, 2003.

[11] "Kabels voor ondergrondse aanleg, met synthetische isolatie en versterkte mantel (type 1kv)," NBN Std. C 33-322, 1975.

[12] C. Gonzalez, J. Geuns, S. Weckx, T. Wijnhoven, P. Vingerhoets, T. De Rybel, and J. Driesen, "LV distribution network feeders in Belgium and power quality issues due to increasing PV penetration levels," in Innovative Smart Grid Technologies (ISGT Europe), 3rd IEEE PES International Conference and Exhibition on. IEEE, 2012, pp. 1-8.

[13] W. Labeeuw and G. Deconinck, "Residential electrical load model based on mixture model clustering and markov models," IEEE Trans. Ind. Informat., to be published.

[14] S. K. Salman and I. M. Rida, "ANN-based AVC relay for voltage control of distribution network with and without embedded generation," in Proc. Int. Conf. Electric Utility Deregulation and Restructuring and Power Technologies DRPT 2000, 2000, pp. 263-267.

[15] K. De Brabandere, B. Bolsens, J. Van den Keybus, A. Woyte, J. Driesen, and R. Belmans, "A voltage and frequency droop control method for parallel inverters," IEEE Transactions on Power Electronics, vol. 22, no. 4, pp. 1107-1115, 2007.

[16] L. Degroote, B. Renders, B. Meersman, and L. Vandevelde, "Neutralpoint shifting and voltage unbalance due to single-phase dg units in low voltage distribution networks," in PowerTech, 2009 IEEE Bucharest. IEEE, 2009, pp. 1-8.

[17] J. Suykens, T. Van Gestel, J. De Brabanter, B. De Moor, and J. Vandewalle, Least Squares Support Vector Machines, World Scientific, Singapore, 2002.

[18] K. Pelckmans, J. Suykens, T. Van Gestel, J. De Brabanter, L. Lukas, B. Hamers, B. De Moor, and J. Vandewalle, "LS-SVMlab: a matlab/c toolbox for least squares support vector machines," Tutorial. KULeuvenESAT. Leuven, Belgium, 2002.

[19] P. McDaniel and S. McLaughlin, "Security and privacy challenges in the smart grid," Security Privacy, IEEE, vol. 7, no. 3, pp. 75 -77, may-june 2009. 